\title{
THE INFLUENCE OF THE MODEL SELECTED PARAMETER MEASUREMENT ERROR ON THE ACCURACY OF HEAT TRANSFER COEFFICIENT DETERMINATION METHOD FOR CONTACT BETWEEN ROLLS AND THE CAST STRAND
}

\begin{abstract}
The paper presents a heat transfer model between two surfaces which are in contact under an external force. The developed heat transfer boundary condition model has been obtained using a new methodology for determining heat flux transferred between two solid surfaces. The methodology consists of two parts: experimental and numerical one. The experimental part involves measurements of temperature at specific points in two samples brought into contact. The numerical part uses an inverse method and the finite element method to calculate the heat flux at the interface.

The effect of uncertainty of input parameters for the calculation of heat transfer coefficient has been analysed. These have included: thermal conductivity, specific heat and location of thermocouples. Verification calculation with new value of input parameters for their impact on the accuracy of the solution has been done.
\end{abstract}

The calculations have been performed using original software which uses the inverse method and FEM.

Keywords: heat transfer, inverse solution, solid to solid interface

\section{Introduction}

Heat transfer is a very important factor in technological processes applied within the steel industry [1-3]. Heat transfer at the solid to solid contact interface is very common. It plays an essential role, especially in metal forming processes and continuous casting of steel. Heat transfer between two solid surfaces is a difficult and complex process. There are many physical phenomena which influence heat flux. Heat transfer occurs by radiation within a very thin gap, and by conduction between two surfaces that are in contact. The modelling of the temperature field, heat flux or heat transfer coefficient and other phenomena as regards the process of heat transfer between two solid surfaces has been analysed by several authors, using both commercial software and original formulations [4-9]. The boundary conditions of heat transfer need to be determined in order to obtain the solution to the heat conduction equation needed to obtain the temperature field. The accuracy of the results of numerical calculations depends on the correct description of the boundary conditions. The heat flux between a tool and a work piece is a function of temperature, pressure and time. For example, in the continuous casting machine, the time of contact between a roll and the cast strand is short but the surface temperature is very high, especially in the first part of the secondary cooling zone. Thus, in this case the heat transfer is intensive. This parameter also strongly depends on the contact pressure. Most of the heat transfer models which are described in literature are related to rolling [10]. In this process high forces are applied so these models are not applicable for heat transfer between the rolls and the surface of the cast strand.
Heat transfer during strand contact with the cooled pull-out and support rolls, which are located in the secondary cooling zone, is difficult to describe. The contact area compared to the strand surface is small. Heat transfer in this case does not significantly influence the cooling of the strand being pulled out. However it causes local changes in the surface temperature (Fig. 1). One can find models describing this effect in literature on the subject. The value of the heat transfer coefficient reaches its maximum of $1500 \mathrm{~W} /\left(\mathrm{m}^{2} \mathrm{~K}\right)$ [11].

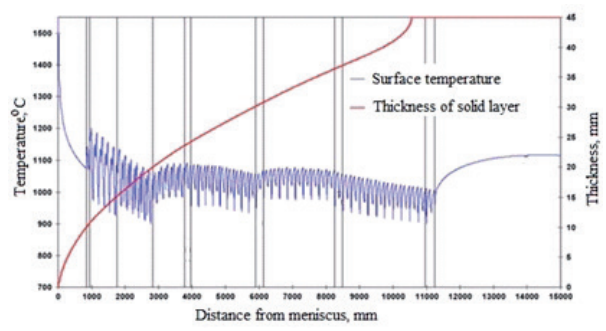

Fig. 1. Changes in the strand surface temperature [12]

This paper presents the methodology for determining the heat transfer combining a physical experiment and numerical methods. The experimental part involves measurements of temperature at specific points of two samples brought into contact. The numerical part uses an inverse method and the finite element method to calculate the heat flux at the interface.

The effect of uncertainty of input parameters for the calculation of heat transfer coefficient was analysed. They included: thermal conductivity, specific heat and location of thermocouples. Calculations with new values of input parameters were performed to verify their impact on the accuracy of the solution.

* AGH UNIVERSITY OF SCIENCE AND TECHNOLOGY FACULTY OF METALS ENGINEERING AND INDUSTRIAL COMPUTER SCIENCE, AL. A. MICKIEWICZA 30, 30-059 KRAKÓW, POLAND

\# Corresponding author: rywotyc@agh.edu.pl 
The methodology for determining the heat transfer coefficient

The schematic diagram of the experimental stand is shown in Fig. 2. In the experiments two samples were used: the Hot sample, which was heated within the furnace, and the Cold sample, which was kept outside the furnace at room temperature. When the Hot sample reached the desired temperature, the furnace has been opened and the sample has been brought into contact with the Cold one at a constant pressure. The material of the Cold sample was WNL steel. The Hot sample was made of C45 steel and was insulated with the ceramic fibre. The Hot sample was heated to an initial temperature of $1000^{\circ} \mathrm{C}$. The samples were brought into contact under a pressure of: $1 \mathrm{MPa}, 10 \mathrm{MPa}, 20 \mathrm{MPa}$ and $30 \mathrm{MPa}$. The time of contact was $30 \mathrm{~s}$ in each test. The full description of the test stand and the experiment is available in the literature [13]

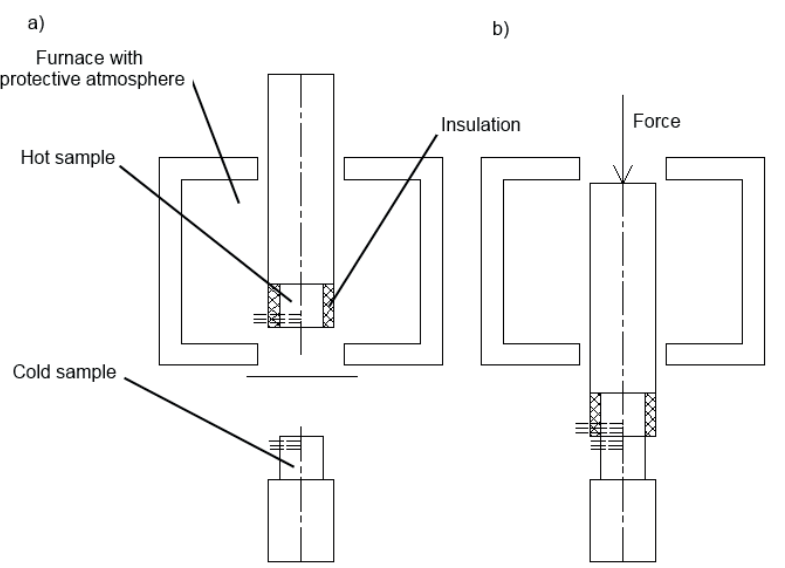

Fig. 2. Schematic diagram of the experiment a) The heating of a sample in the furnace; $b$ ) The positions of samples during the test

a)

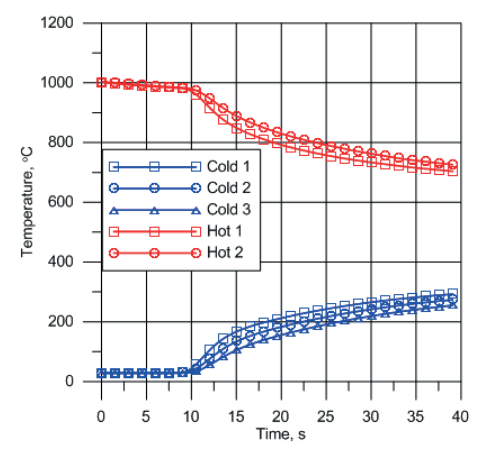

c)

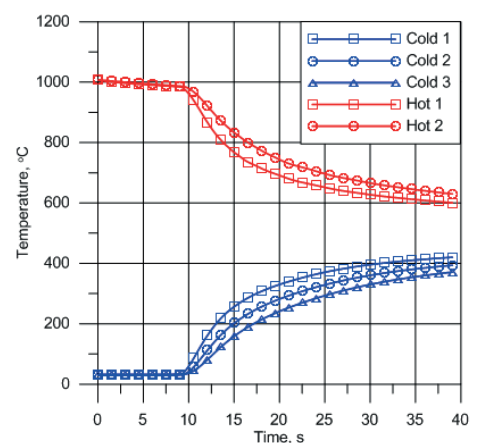

The results of the temperature measurements are shown in Fig. 3. Increasing the pressure leads to a higher temperature in the Cold sample and to a lower temperature in the Hot sample for each tests.

The numerical model consisted of the inverse method employed to calculate heat flux at the interface between the Cold and the Hot sample. The heat flux at the interface was determined from the solution to the boundary inverse heat conduction problem. The boundary condition of the heat flux resulted from temperature variations at points located inside the Cold and Hot samples. The computational algorithm for solving the inverse problem for cooling a three dimensional plate was given by Malinowski et al. [14]. The necessary modification resulting from a reduction of a three dimensional heat conduction problem to an axially symmetrical one will be given. The calculation results that are presented in Fig. 4 allow determining changes in heat fluxes transferred between the samples at various pressures.

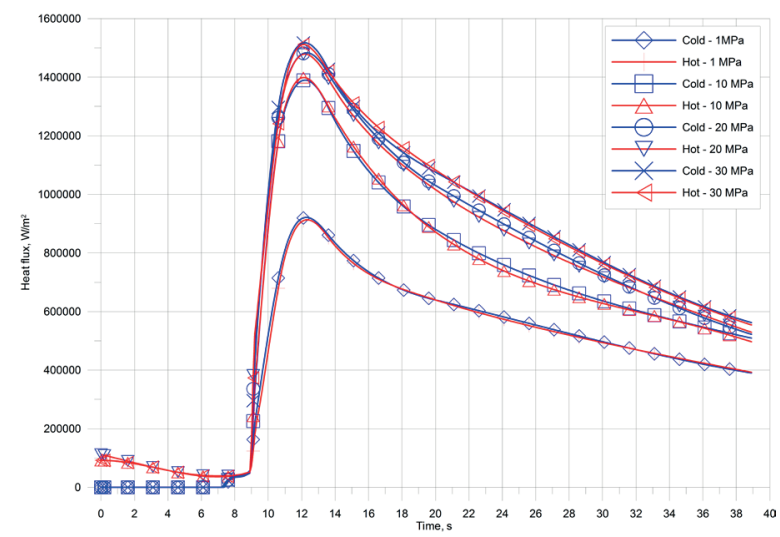

Fig. 4. The heat flux as a function of time for an initial temperature of $1000^{\circ} \mathrm{C}$

b)

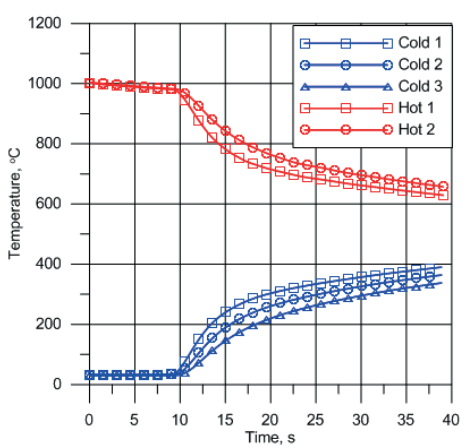

d)

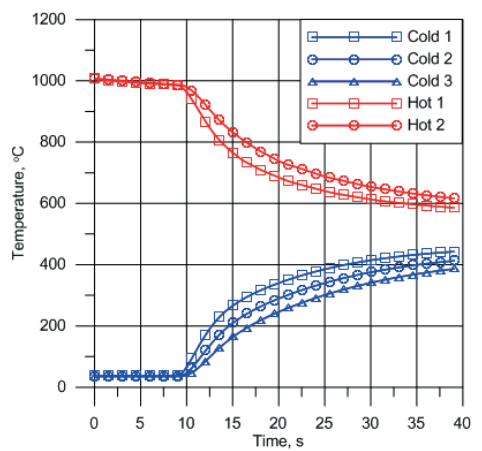

Fig. 3. The temperature distributions vs. time for the initial sample temperature of $1000{ }^{\circ} \mathrm{C}$ and the pressure of : a) $\left.1 \mathrm{MPa}, \mathrm{b}\right) 10 \mathrm{MPa}$, c) 20 $\mathrm{MPa}$ d) $30 \mathrm{MPa}$ 


\section{Analysis of the effect of the thermo-physical property measurement error}

On the basis of the obtained solution (Fig. 4), third degree polynomials were applied to determine changes in the value of the heat transfer coefficient over time for the contact surfaces. The locations of nodes and the values of polynomial coefficients are presented in Table 1.

The temperature was calculated at points corresponding to the location of thermocouples in both samples, using a previously determined function to approximate the heat transfer coefficient. The obtained temperature changes were used for a series of calculations of the influence of the selected parameters on the value of the heat transfer coefficient.

The errors of all physical parameters used for the solution impacted the searched parameter. Heat conductivity and specific heat, as well as the location of thermocouples in the samples were analysed. Test calculations were performed, in

TABLE 1

Third degree polynomial coefficients

\begin{tabular}{|c|c|c|}
\hline \multicolumn{3}{|r|}{$\mathrm{p}=1 \mathrm{MPa}$} \\
\hline Time interval, $\mathrm{s}$ & Sample & Function \\
\hline $7.5-8.9$ & Cold & $2.892587 \mathrm{E}+01 \mathrm{x}^{3}-7.152491 \mathrm{E}+02 \mathrm{x}^{2}+5.902682 \mathrm{E}+03 \mathrm{x}-1.621779 \mathrm{E}+04$ \\
\hline $0-8.9$ & Hot & $3.586807 \mathrm{E}-01 \mathrm{x}^{3}-3.809130 \mathrm{E}+00 \mathrm{x}^{2}+9.759748 \mathrm{E}-01 \mathrm{x}+9.461152 \mathrm{E}+01$ \\
\hline \multirow{2}{*}{$8.9-9.2$} & Cold & $-1.899333 \mathrm{E}+04 \mathrm{x}^{3}+5.171120 \mathrm{E}+05 \mathrm{x}^{2}-4.691163 \mathrm{E}+06 \mathrm{x}+1.418067 \mathrm{E}+07$ \\
\hline & Hot & $-3.623333 \mathrm{E}+03 \mathrm{x}^{3}+9.928450 \mathrm{E}+04 \mathrm{x}^{2}-9.062330 \mathrm{E}+05 \mathrm{x}+2.755541 \mathrm{E}+06$ \\
\hline \multirow{2}{*}{$9.2-11.0$} & Cold & $-3.360673 \mathrm{E}+01 \mathrm{x}^{3}+8.725360 \mathrm{E}+02 \mathrm{x}^{2}-6.610436 \mathrm{E}+03 \mathrm{x}+1.358656 \mathrm{E}+04$ \\
\hline & Hot & $-4.645937 E+01 x^{3}+1.405250 E+03 x^{2}-1.374062 E+04 x+4.384823 E+04$ \\
\hline \multirow{2}{*}{$11.0-13.5$} & Cold & $8.643322 \mathrm{E}+00 \mathrm{x}^{3}-4.092892 \mathrm{E}+02 \mathrm{x}^{2}+6.252943 \mathrm{E}+03 \mathrm{x}-2.904459 \mathrm{E}+04$ \\
\hline & Hot & $1.216066 \mathrm{E}+01 \mathrm{x}^{3}-5.195822 \mathrm{E}+02 \mathrm{x}^{2}+7.310831 \mathrm{E}+03 \mathrm{x}-3.283779 \mathrm{E}+04$ \\
\hline \multirow{2}{*}{$13.5-20.0$} & Cold & $-3.179481 \mathrm{E}-01 \mathrm{x}^{3}+1.976073 \mathrm{E}+01 \mathrm{x}^{2}-4.318388 \mathrm{E}+02 \mathrm{x}+5.053699 \mathrm{E}+03$ \\
\hline & Hot & $-6.096642 \mathrm{E}-01 \mathrm{x}^{3}+3.469900 \mathrm{E}+01 \mathrm{x}^{2}-6.725893 \mathrm{E}+02 \mathrm{x}+5.340435 \mathrm{E}+03$ \\
\hline \multirow{2}{*}{$20.0-39.0$} & Cold & $5.830742 \mathrm{E}-02 \mathrm{x}^{3}-5.349578 \mathrm{E}+00 \mathrm{x}^{2}+1.210665 \mathrm{E}+02 \mathrm{x}+1.029669 \mathrm{E}+03$ \\
\hline & Hot & $-2.041499 \mathrm{E}-03 \mathrm{x}^{3}+2.261530 \mathrm{E}-01 \mathrm{x}^{2}-2.282349 \mathrm{E}+01 \mathrm{x}+1.273273 \mathrm{E}+03$ \\
\hline \multicolumn{3}{|r|}{$\mathrm{p}=10 \mathrm{MPa}$} \\
\hline $7.5-8.9$ & Cold & $3.602339 \mathrm{E}+01 \mathrm{x}^{3}-8.848533 \mathrm{E}+02 \mathrm{x}^{2}+7.251646 \mathrm{E}+03 \mathrm{x}-1.978884 \mathrm{E}+04$ \\
\hline $0-8.9$ & Hot & $1.383650 \mathrm{E}-01 \mathrm{x}^{3}-4.992635 \mathrm{E}-01 \mathrm{x}^{2}-1.404186 \mathrm{E}+01 \mathrm{x}+1.156568 \mathrm{E}+02$ \\
\hline \multirow{2}{*}{$8.9-9.2$} & Cold & $-1.115500 \mathrm{E}+04 \mathrm{x}^{3}+3.041570 \mathrm{E}+05 \mathrm{x}^{2}-2.763170 \mathrm{E}+06 \mathrm{x}+8.363927 \mathrm{E}+06$ \\
\hline & Hot & $-1.552833 \mathrm{E}+04 \mathrm{x}^{3}+4.228495 \mathrm{E}+05 \mathrm{x}^{2}-3.836713 \mathrm{E}+06 \mathrm{x}+1.159988 \mathrm{E}+07$ \\
\hline \multirow{2}{*}{$9.2-11.0$} & Cold & $-4.792613 \mathrm{E}+01 \mathrm{x}^{3}+1.310304 \mathrm{E}+03 \mathrm{x}^{2}-1.109944 \mathrm{E}+04 \mathrm{x}+2.887308 \mathrm{E}+04$ \\
\hline & Hot & $-6.217383 E+01 x^{3}+1.775999 E+03 x^{2}-1.612827 E+04 x+4.684783 E+04$ \\
\hline \multirow{2}{*}{$11.0-13.5$} & Cold & $3.899038 \mathrm{E}+00 \mathrm{x}^{3}-2.301083 \mathrm{E}+02 \mathrm{x}^{2}+3.977094 \mathrm{E}+03 \mathrm{x}-1.955818 \mathrm{E}+04$ \\
\hline & Hot & $9.923660 \mathrm{E}+00 \mathrm{x}^{3}-4.568304 \mathrm{E}+02 \mathrm{x}^{2}+6.822571 \mathrm{E}+03 \mathrm{x}-3.140086 \mathrm{E}+04$ \\
\hline \multirow{2}{*}{$13.5-20.0$} & Cold & $-1.764276 \mathrm{E}-01 \mathrm{x}^{3}+1.375694 \mathrm{E}+01 \mathrm{x}^{2}-3.790043 \mathrm{E}+02 \mathrm{x}+4.831892 \mathrm{E}+03$ \\
\hline & Hot & $1.772250 \mathrm{E}-01 \mathrm{x}^{3}-6.409348 \mathrm{E}+00 \mathrm{x}^{2}-9.914148 \mathrm{E}+00 \mathrm{x}+2.728338 \mathrm{E}+03$ \\
\hline \multirow{2}{*}{$20.0-39.0$} & Cold & $-2.973535 \mathrm{E}-02 \mathrm{x}^{3}+3.181536 \mathrm{E}+00 \mathrm{x}^{2}-1.320169 \mathrm{E}+02 \mathrm{x}+2.948769 \mathrm{E}+03$ \\
\hline & Hot & $-8.226718 \mathrm{E}-02 \mathrm{x}^{3}+7.904865 \mathrm{E}+00 \mathrm{x}^{2}-2.710965 \mathrm{E}+02 \mathrm{x}+4.302233 \mathrm{E}+03$ \\
\hline \multicolumn{3}{|r|}{$\mathrm{p}=20 \mathrm{MPa}$} \\
\hline $7.5-8.9$ & Cold & $2.252451 \mathrm{E}+01 \mathrm{x}^{3}-5.644052 \mathrm{E}+02 \mathrm{x}^{2}+4.720125 \mathrm{E}+03 \mathrm{x}-1.313337 \mathrm{E}+04$ \\
\hline $0-8.9$ & Hot & $1.658828 \mathrm{E}-01 \mathrm{x}^{3}-8.092168 \mathrm{E}-01 \mathrm{x}^{2}-1.311261 \mathrm{E}+01 \mathrm{x}+1.150785 \mathrm{E}+02$ \\
\hline \multirow{2}{*}{$8.9-9.2$} & Cold & $-2.466333 E+04 x^{3}+6.707970 E+05 x^{2}-6.079413 E+06 x+1.835988 E+07$ \\
\hline & Hot & $-3.132833 E+04 x^{3}+8.514670 E+05 x^{2}-7.711549 E+06 x+2.327364 E+07$ \\
\hline
\end{tabular}




\begin{tabular}{|c|c|c|}
\hline \multirow{2}{*}{$9.2-11.0$} & Cold & $-5.940485 E+01 x^{3}+1.695222 E+03 x^{2}-1.536929 E+04 x+4.466767 E+04$ \\
\hline & Hot & $-8.675779 \mathrm{E}+01 \mathrm{x}^{3}+2.586770 \mathrm{E}+03 \mathrm{x}^{2}-2.501346 \mathrm{E}+04 \mathrm{x}+7.929574 \mathrm{E}+04$ \\
\hline \multirow{2}{*}{$11.0-13.5$} & Cold & $6.491741 \mathrm{E}+00 \mathrm{x}^{3}-3.213558 \mathrm{E}+02 \mathrm{x}^{2}+5.074953 \mathrm{E}+03 \mathrm{x}-2.392144 \mathrm{E}+04$ \\
\hline & Hot & $8.357496 \mathrm{E}+00 \mathrm{x}^{3}-4.016758 \mathrm{E}+02 \mathrm{x}^{2}+6.205528 \mathrm{E}+03 \mathrm{x}-2.910956 \mathrm{E}+04$ \\
\hline \multirow{2}{*}{$13.5-20.0$} & Cold & $3.846285 \mathrm{E}-01 \mathrm{x}^{3}-1.772283 \mathrm{E}+01 \mathrm{x}^{2}+2.159181 \mathrm{E}+02 \mathrm{x}+1.364206 \mathrm{E}+03$ \\
\hline & Hot & $-2.062628 \mathrm{E}-01 \mathrm{x}^{3}+1.248080 \mathrm{E}+01 \mathrm{x}^{2}-2.944607 \mathrm{E}+02 \mathrm{x}+4.230306 \mathrm{E}+03$ \\
\hline \multirow{2}{*}{$20.0-39.0$} & Cold & $2.988207 \mathrm{E}-02 \mathrm{x}^{3}-2.676059 \mathrm{E}+00 \mathrm{x}^{2}+3.974500 \mathrm{E}+01 \mathrm{x}+1.706933 \mathrm{E}+03$ \\
\hline & Hot & $-9.648267 \mathrm{E}-03 \mathrm{x}^{3}+1.068179 \mathrm{E}+00 \mathrm{x}^{2}-7.389390 \mathrm{E}+01 \mathrm{x}+2.811101 \mathrm{E}+03$ \\
\hline \multicolumn{3}{|r|}{$\mathrm{p}=30 \mathrm{MPa}$} \\
\hline $7.5-8.9$ & Cold & $2.892587 \mathrm{E}+01 \mathrm{x}^{3}-7.152491 \mathrm{E}+02 \mathrm{x}^{2}+5.902682 \mathrm{E}+03 \mathrm{x}-1.621779 \mathrm{E}+04$ \\
\hline $0-8.9$ & Hot & $1.721498 \mathrm{E}-01 \mathrm{x}^{3}-8.545648 \mathrm{E}-01 \mathrm{x}^{2}-1.314945 \mathrm{E}+01 \mathrm{x}+1.154999 \mathrm{E}+02$ \\
\hline \multirow{2}{*}{$8.9-9.2$} & Cold & $-1.899331 \mathrm{E}+04 \mathrm{x}^{3}+5.171121 \mathrm{E}+05 \mathrm{x}^{2}-4.691162 \mathrm{E}+06 \mathrm{x}+1.418068 \mathrm{E}+07$ \\
\hline & Hot & $-2.908833 \mathrm{E}+04 \mathrm{x}^{3}+7.908320 \mathrm{E}+05 \mathrm{x}^{2}-7.164536 \mathrm{E}+06 \mathrm{x}+2.162900 \mathrm{E}+07$ \\
\hline \multirow{2}{*}{$9.2-11.0$} & Cold & $-3.360673 E+01 x^{3}+8.725360 E+02 x^{2}-6.610436 E+03 x+1.358656 E+04$ \\
\hline & Hot & $-3.514249 \mathrm{E}+01 \mathrm{x}^{3}+1.018158 \mathrm{E}+03 \mathrm{x}^{2}-9.147933 \mathrm{E}+03 \mathrm{x}+2.589524 \mathrm{E}+04$ \\
\hline \multirow{2}{*}{$11.0-13.5$} & Cold & $8.643322 E+00 x^{3}-4.092892 E+02 x^{2}+6.252943 E+03 x-2.904459 E+04$ \\
\hline & Hot & $2.292787 \mathrm{E}+01 \mathrm{x}^{3}-9.505271 \mathrm{E}+02 \mathrm{x}^{2}+1.308358 \mathrm{E}+04 \mathrm{x}-5.773213 \mathrm{E}+04$ \\
\hline \multirow{2}{*}{$13.5-20.0$} & Cold & $-3.179481 \mathrm{E}-01 \mathrm{x}^{3}+1.976073 \mathrm{E}+01 \mathrm{x}^{2}-4.318388 \mathrm{E}+02 \mathrm{x}+5.053699 \mathrm{E}+03$ \\
\hline & Hot & $-1.263000 \mathrm{E}-01 \mathrm{x}^{3}+7.191573 \mathrm{E}+00 \mathrm{x}^{2}-1.699360 \mathrm{E}+02 \mathrm{x}+3.368018 \mathrm{E}+03$ \\
\hline \multirow{2}{*}{$20.0-39.0$} & Cold & $5.830742 \mathrm{E}-02 \mathrm{x}^{3}-5.349578 \mathrm{E}+00 \mathrm{x}^{2}+1.210665 \mathrm{E}+02 \mathrm{x}+1.029669 \mathrm{E}+03$ \\
\hline & Hot & $3.836555 \mathrm{E}-02 \mathrm{x}^{3}-3.391136 \mathrm{E}+00 \mathrm{x}^{2}+5.577033 \mathrm{E}+01 \mathrm{x}+1.769649 \mathrm{E}+03$ \\
\hline
\end{tabular}

Calculation variants and average error of temperature calculations - thermal properties

\begin{tabular}{|c|c|c|c|c|c|c|c|}
\hline \multirow{2}{*}{$\begin{array}{c}\text { Test } \\
\text { symbol }\end{array}$} & \multirow{2}{*}{$\begin{array}{l}\text { Change in the thermal } \\
\text { conductivity coefficient }\end{array}$} & \multirow{2}{*}{$\begin{array}{c}\text { Change in the specific } \\
\text { heat }\end{array}$} & \multirow{2}{*}{ Sample } & \multicolumn{4}{|c|}{ Relative average error of temperature calculations, $\%$} \\
\hline & & & & $1 \mathrm{MPa}$ & $10 \mathrm{MPa}$ & $20 \mathrm{MPa}$ & $30 \mathrm{MPa}$ \\
\hline T0 & $0 \%$ & $0 \%$ & - & - & - & - & - \\
\hline \multirow{2}{*}{$\mathrm{C} 1$} & \multirow{2}{*}{$-10 \%$} & \multirow{2}{*}{$0 \%$} & Cold & 0.90 & 1,25 & 1,40 & 1,44 \\
\hline & & & Hot & 1.09 & 1.43 & 1.57 & 1.62 \\
\hline \multirow{2}{*}{$\mathrm{C} 2$} & \multirow{2}{*}{$-5 \%$} & \multirow{2}{*}{$0 \%$} & Cold & 0,43 & 0,60 & 0,67 & 0,70 \\
\hline & & & Hot & 0.53 & 0.69 & 0.76 & 0.78 \\
\hline \multirow{2}{*}{$\mathrm{C} 3$} & \multirow{2}{*}{$+5 \%$} & \multirow{2}{*}{$0 \%$} & Cold & 0,40 & 0,56 & 0,63 & 0,64 \\
\hline & & & Hot & 0.49 & 0.64 & 0.71 & 0.74 \\
\hline \multirow{2}{*}{$\mathrm{C} 4$} & \multirow{2}{*}{$+10 \%$} & \multirow{2}{*}{$0 \%$} & Cold & 0,78 & 1,09 & 1,22 & 1,25 \\
\hline & & & Hot & 0.95 & 1.24 & 1.37 & 1.43 \\
\hline \multirow{2}{*}{ H1 } & \multirow{2}{*}{$0 \%$} & \multirow{2}{*}{$-10 \%$} & Cold & 0.83 & 1.14 & 1.27 & 1.31 \\
\hline & & & Hot & 1.29 & 1.58 & 1.71 & 1.77 \\
\hline \multirow{2}{*}{$\mathrm{H} 2$} & \multirow{2}{*}{$0 \%$} & \multirow{2}{*}{$-5 \%$} & Cold & 0.41 & 0.56 & 0.63 & 0.64 \\
\hline & & & Hot & 0.64 & 0.78 & 0.84 & 0.87 \\
\hline \multirow{2}{*}{ H3 } & \multirow{2}{*}{$0 \%$} & \multirow{2}{*}{$+5 \%$} & Cold & 0.40 & 0.55 & 0.61 & 0.64 \\
\hline & & & Hot & 0.62 & 0.75 & 0.82 & 0.84 \\
\hline \multirow{2}{*}{$\mathrm{H} 4$} & \multirow{2}{*}{$0 \%$} & \multirow{2}{*}{$+10 \%$} & Cold & 0.78 & 1.08 & 1.21 & 1.25 \\
\hline & & & Hot & 1.22 & 1.49 & 1.62 & 1.66 \\
\hline
\end{tabular}


which measurement errors of these parameters were simulated and their influence on the result of numerical calculations was analysed.

The calculation results for tests with errors were compared with the results obtained for the master test T0. Errors in the form of the relative heat transfer coefficient calculation error and the average temperature calculation error for points corresponding to the location of thermocouples during the test were determined.

The thermo-physical properties for both samples were expressed as functions of temperature (Fig. 5 and Fig. 6) $[12,15]$. Calculations for these parameters were the basis for comparisons and the analysis.

The first series of calculations was conducted in order to analyse the effect of the determination error of thermal conductivity for materials applied in the experiment. This error was assumed within the limits of $5 \%$ and $10 \%$. The second calculation series aimed at analysing the specific heat measurement error. This error also was assumed within the limits of $5 \%$ and $10 \%$. The comparison of calculation variants is presented in table 2 .

The error of heat transfer coefficient calculations concerning the analysed changes of thermo-physical parameters is presented in Fig. 7 and Fig. 8, whereas the average error of temperature calculations is presented in Table 2. The error of determination of the heat transfer coefficient in the analysed range is proportional to the measurement error of the specific heat and heat transfer coefficient. It slightly exceeds $5 \%$ for thermal conductivity and $15 \%$ for specific heat. The biggest error for the change of specific heat arises from the fact that for the same value of relative error the specific heat measurement error is bigger than for the heat conductivity coefficient. It is also relevant how the accuracy of determining the thermo-physical parameters influences the temperature field determined with the result obtained from the inverse solution. The data presented in Table 3 illustrates that this influence is not significant.
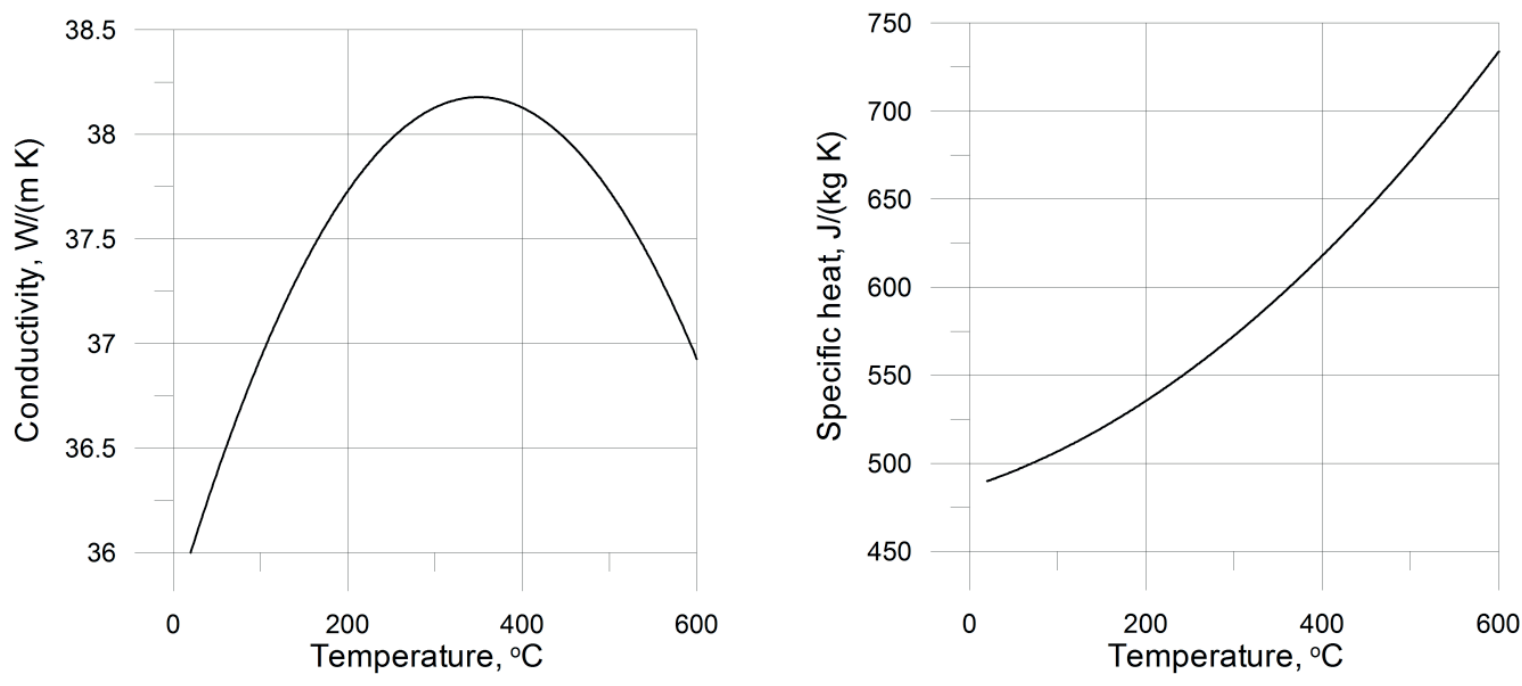

Fig. 5. Conductivity and specific heat for the Cold sample
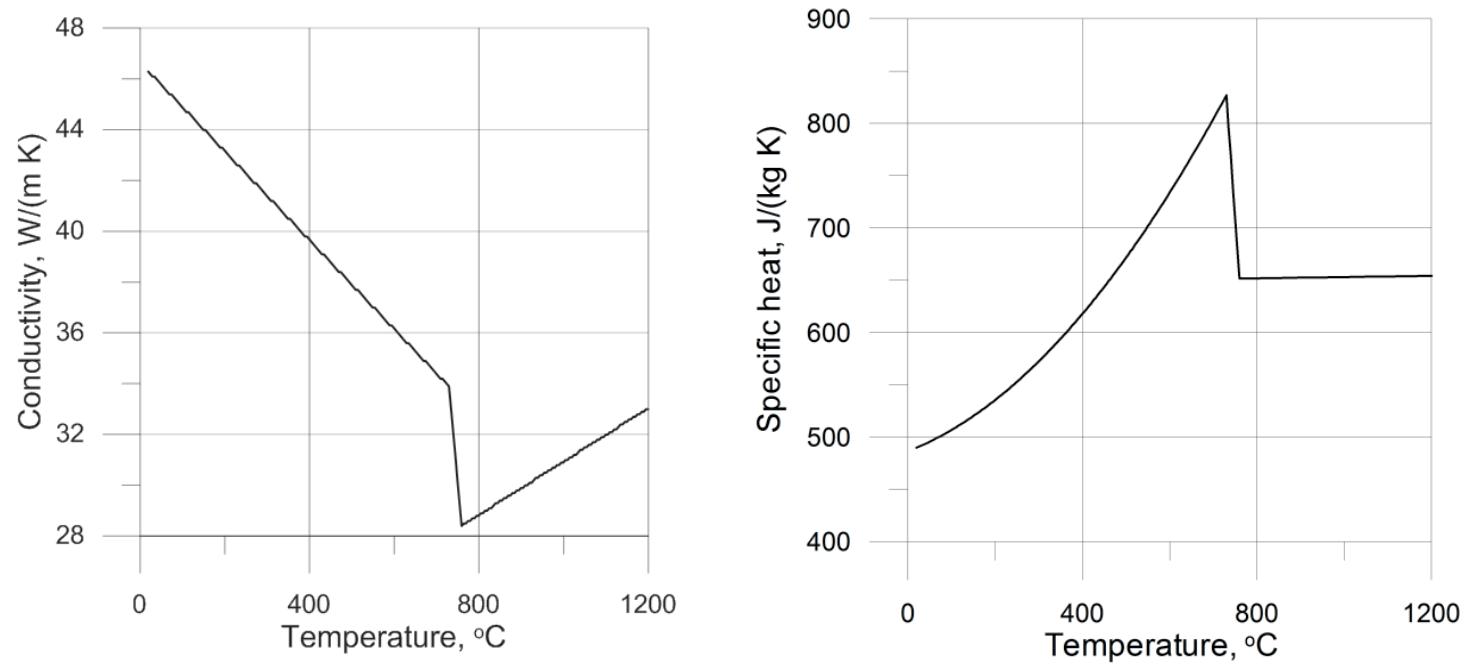

Fig. 6. Conductivity and specific heat for the Hot sample 
a)

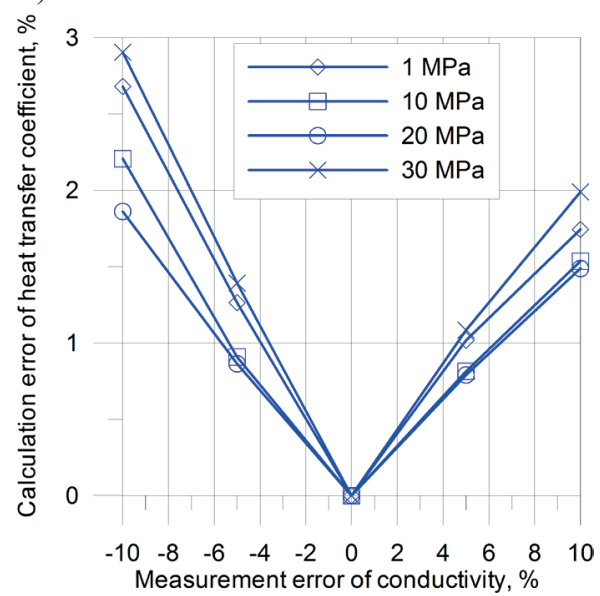

b)

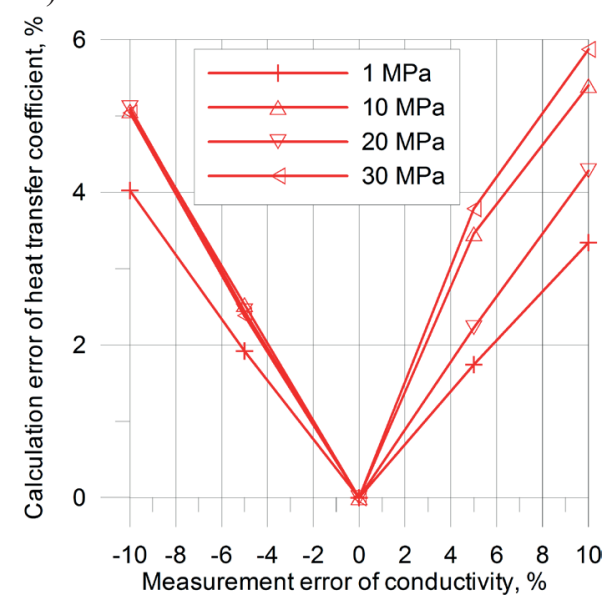

Fig 7. The impact of heat conductivity measurement error on the heat transfer coefficient (tests C1- C4) a - Cold sample, b - Hot sample

a)

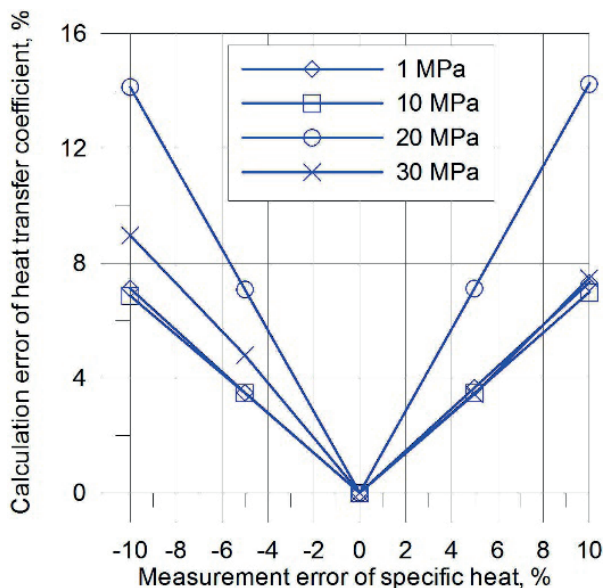

b)

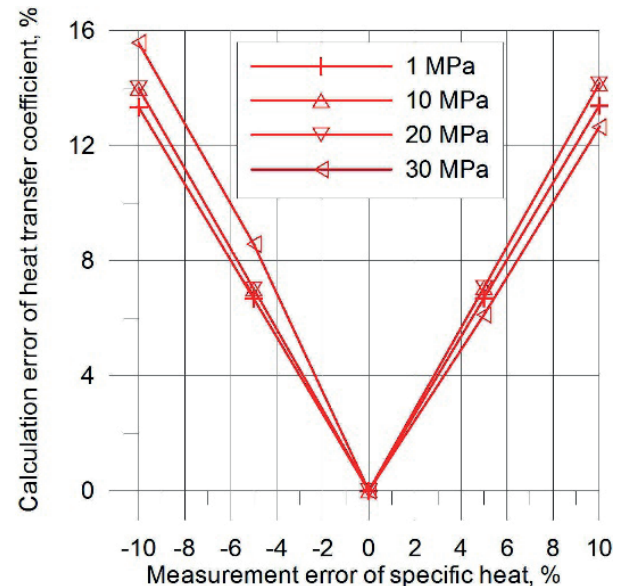

Fig 8 . The impact of specific heat measurement error on the heat transfer coefficient. (tests H1- H4) a - Cold sample, b - Hot sample

a)

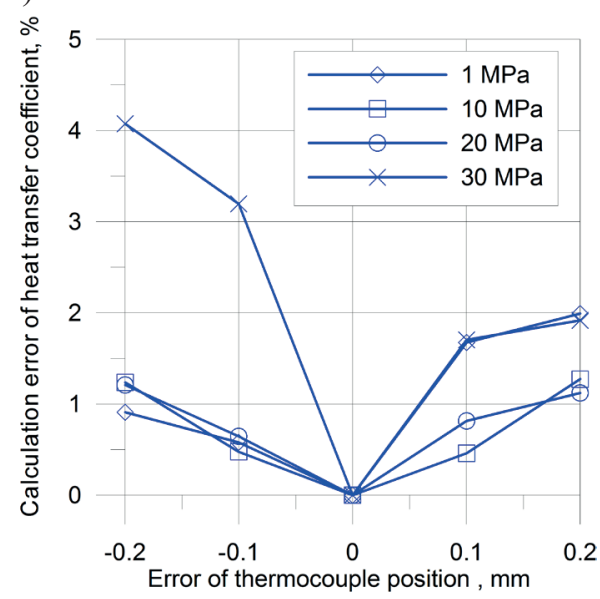

b)

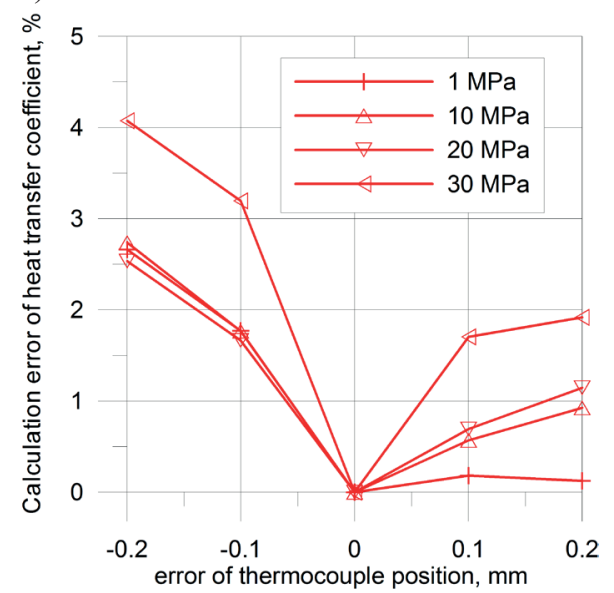

Fig 9. Results of calculation for test A1 - A4, a - Cold sample b - Hot sample

The second series of calculations was conducted in order to determine the influence of error of thermocouple installation in samples. A case was considered, in which all thermocouples were shifted by $0.1 \mathrm{~mm}$ and $0.2 \mathrm{~mm}$. Also series of tests, in which only 1 thermocouple was incorrectly installed, were performed. This error was also $0.1 \mathrm{~mm}$ and $0.2 \mathrm{~mm}$. Table 3 presents all calculation variants in this series. The error of heat transfer coefficient calculations concerning the analysed changes of position of thermocouple is presented in Fig. 9 - Fig. 12. 
TABLE 3

Variants and results of calculations - thermocouple location error

\begin{tabular}{|c|c|c|c|c|c|c|c|c|}
\hline \multirow{2}{*}{$\begin{array}{l}\text { Test } \\
\text { symbol }\end{array}$} & \multirow{2}{*}{$\begin{array}{c}\text { Change in } \\
\text { thermocouple } 1 \\
\text { location }\end{array}$} & \multirow{2}{*}{$\begin{array}{c}\text { Change in } \\
\text { thermocouple } 2 \\
\text { location }\end{array}$} & \multirow{2}{*}{$\begin{array}{c}\text { Change in } \\
\text { thermocouple } 3 \\
\text { location }\end{array}$} & \multirow{2}{*}{ Sample } & \multicolumn{4}{|c|}{$\begin{array}{c}\text { Relative average error of temperature calculations, } \\
\%\end{array}$} \\
\hline & & & & & $1 \mathrm{MPa}$ & $10 \mathrm{MPa}$ & $20 \mathrm{MPa}$ & $30 \mathrm{MPa}$ \\
\hline \multirow{2}{*}{ A1 } & \multirow{2}{*}{$-0,2$} & \multirow{2}{*}{0,0} & \multirow{2}{*}{0,0} & Cold & 0.06 & 0.09 & 0.10 & 0.10 \\
\hline & & & & Hot & 1.09 & 0.77 & 0.66 & 0.62 \\
\hline \multirow{2}{*}{ A2 } & \multirow{2}{*}{$-0,1$} & \multirow{2}{*}{0,0} & \multirow{2}{*}{0,0} & Cold & 0.03 & 0.04 & 0.05 & 0.05 \\
\hline & & & & Hot & 0.57 & 0.40 & 0.34 & 0.32 \\
\hline \multirow{2}{*}{ A3 } & \multirow{2}{*}{0,1} & \multirow{2}{*}{0,0} & \multirow{2}{*}{0,0} & Cold & 0.03 & 0.04 & 0.05 & 0.06 \\
\hline & & & & Hot & 0.15 & 0.26 & 0.30 & 0.32 \\
\hline \multirow{2}{*}{ A4 } & \multirow{2}{*}{0,2} & \multirow{2}{*}{0,0} & \multirow{2}{*}{0,0} & Cold & 0.06 & 0.09 & 0.10 & 0.10 \\
\hline & & & & Hot & 0.28 & 0.50 & 0.57 & 0.61 \\
\hline \multirow[t]{2}{*}{ A5 } & \multirow{2}{*}{0,0} & \multirow{2}{*}{$-0,2$} & \multirow{2}{*}{0,0} & Cold & 0.80 & 1.10 & 1.24 & 1.29 \\
\hline & & & & Hot & 1.27 & 0.92 & 0.81 & 0.77 \\
\hline \multirow[t]{2}{*}{ A6 } & \multirow{2}{*}{0,0} & 01 & 0 & Cold & 0.40 & 0.55 & 0.62 & 0.65 \\
\hline & & $-0,1$ & 0,0 & Hot & 0.68 & 0.49 & 0.43 & 0.41 \\
\hline A7 & O & 01 & 00 & Cold & 0.40 & 0.56 & 0.63 & 0.65 \\
\hline At & 0,0 & 0,1 & 0 & Hot & 0.58 & 0.48 & 0.43 & 0.41 \\
\hline 19 & م0 & ? & 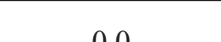 & Cold & 0.81 & 1.12 & 1.26 & 1.31 \\
\hline A & 0,0 & 0,2 & 0,0 & Hot & 1.07 & 0.82 & 0.71 & 0.67 \\
\hline A9 & 00 & 00 & -02 & Cold & 0.73 & 1.01 & 1.14 & 1.19 \\
\hline A9 & 0,0 & 0,0 & $-0,2$ & Hot & 0.16 & 0.15 & 0.15 & 0.15 \\
\hline$\Delta 10$ & 0 & 00 & 0 & Cold & 0.37 & 0.51 & 0.57 & 0.60 \\
\hline AIV & 0,0 & 0,0 & $-0,1$ & Hot & 0.09 & 0.09 & 0.08 & 0.08 \\
\hline$\Delta$ & r & 00 & P & Cold & 0.37 & 0.51 & 0.58 & 0.59 \\
\hline A11 & 0,0 & 0,0 & 0,1 & Hot & 0.16 & 0.31 & 0.37 & 0.40 \\
\hline$\Delta 12$ & 0 & 00 & 02 & Cold & 0.74 & 1.03 & 1.16 & 1.20 \\
\hline A12 & 0,0 & 0,0 & 0,2 & Hot & 0.28 & 0.58 & 0.70 & 0.76 \\
\hline A13 & -01 & -01 & -01 & Cold & 0.06 & 0.09 & 0.10 & 0.10 \\
\hline & & & & Hot & 1.09 & 0.77 & 0.66 & 0.62 \\
\hline A14 & ? & 0 & ? & Cold & 0.03 & 0.04 & 0.05 & 0.05 \\
\hline A14 & $-0,2$ & $-0,2$ & $-0,2$ & Hot & 0.57 & 0.40 & 0.34 & 0.32 \\
\hline A15 & 01 & 01 & 01 & Cold & 0.03 & 0.04 & 0.05 & 0.06 \\
\hline תונ & 0,1 & 0,1 & 0,1 & Hot & 0.15 & 0.26 & 0.30 & 0.32 \\
\hline A16 & 02 & 02 & 02 & Cold & 0.06 & 0.09 & 0.10 & 0.10 \\
\hline & 0,2 & 0,2 & 0,2 & Hot & 0.28 & 0.50 & 0.57 & 0.61 \\
\hline
\end{tabular}

a)

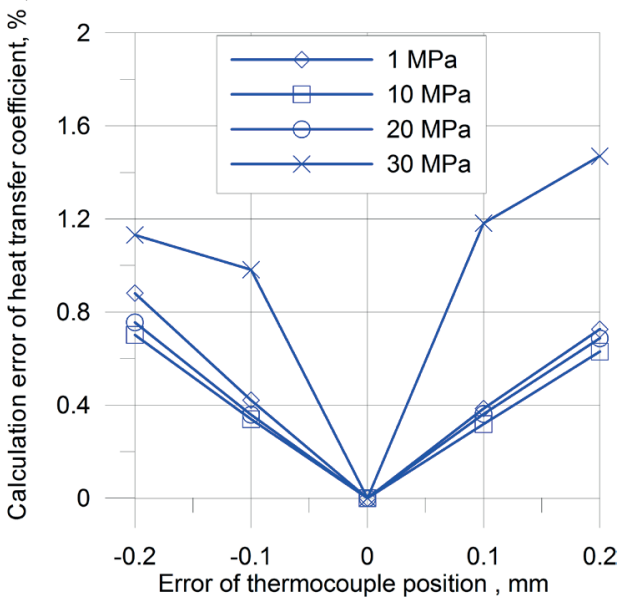

b)

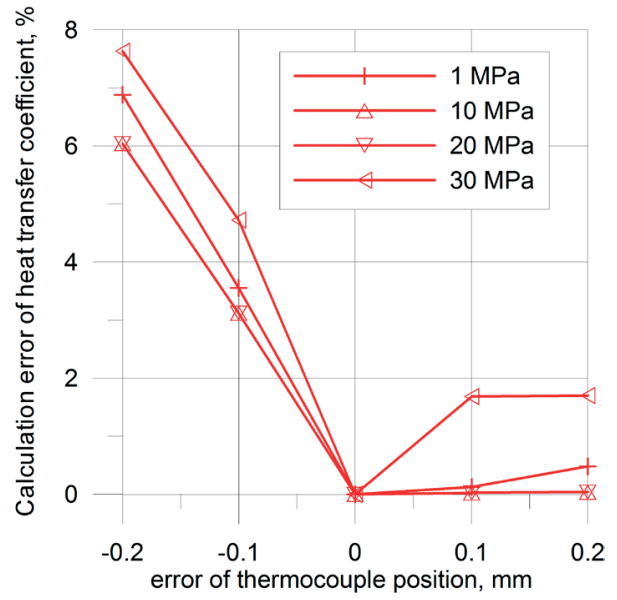

Fig. 10. Results of calculation for test A5 - A8, a - Cold sample b-Hot sample 
a)

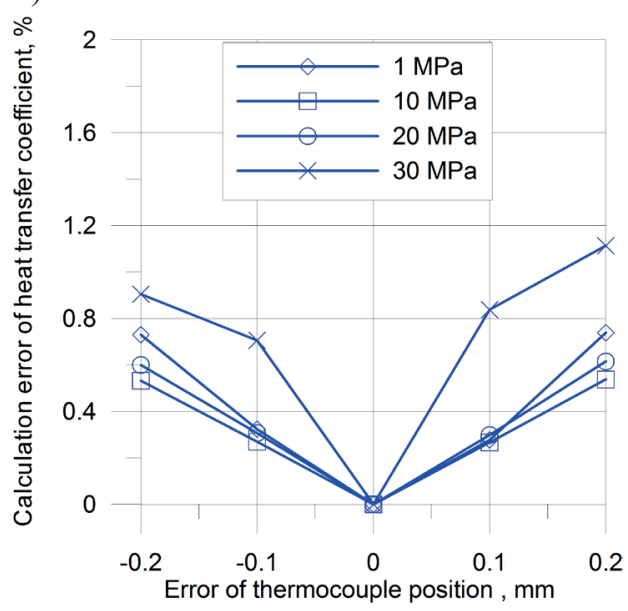

b)

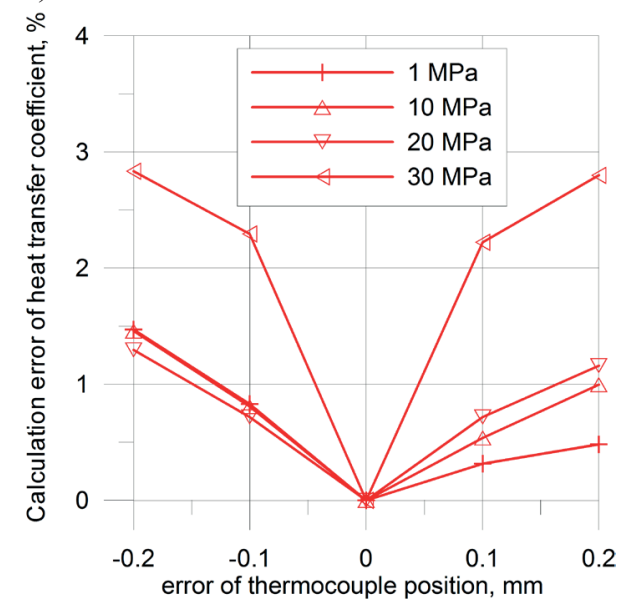

Fig. 11. Results of calculation for test A9 - A12, a - Cold sample b - Hot sample

a)

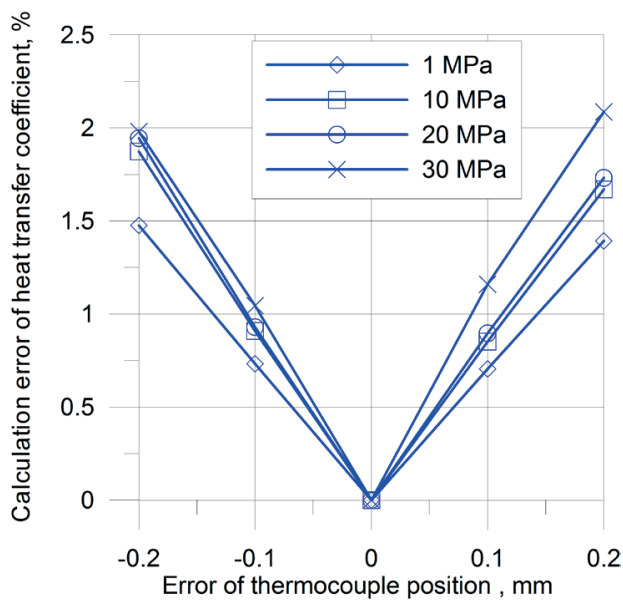

b)

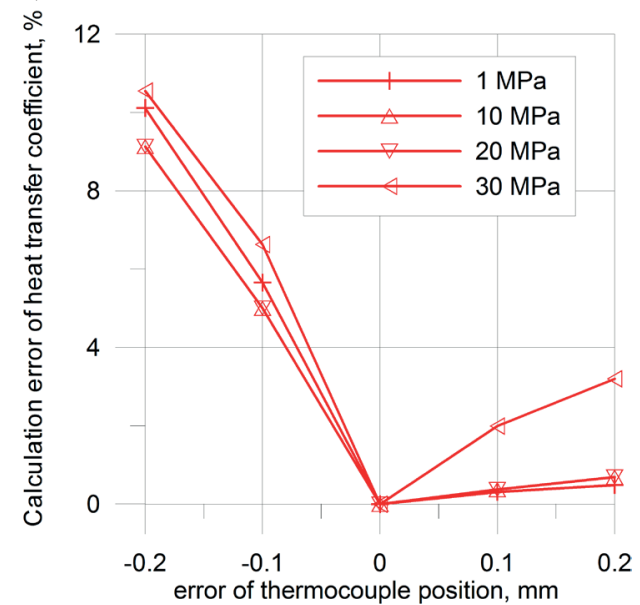

Fig. 12. Results of calculation for test A13 - A16 a - Cold sample b- Hot sample

Within the range of assumed values, the calculation error of the heat transfer coefficient is proportional to the error of the thermocouple installation in the samples. A shift of the points towards the surface for the Cold sample makes the heat transfer coefficient value underrated. For the Hot sample, when the measurement points are shifted towards the surface, the values obtained from the inverse problem solution are overrated.

In both cases the errors do not exceed $10 \%$, which shows a small sensitivity of the method to inaccuracies in the thermocouple installation in the samples that were used in the experiment

\section{Conclusion}

The paper presents a model of heat transfer between two solid faces being in contact as a result of the force applied. The value of the heat transfer coefficient was obtained from original research using a new methodology for determining the heat flux between these faces. It consists of two stages: an experiment and numerical computations. The impact of the measurement error of the parameters set in the inverse solution on the computation result of the heat transfer coefficient was analysed. The parameters were: thermal conductivity, specific heat and location of measurement thermocouples. Test calculations, in which measurement errors of these parameters were simulated and their influence on the accuracy of the obtained solution was analysed, were conducted.

Test calculations of the heat transfer coefficient from the inverse solutions were carried out, where measurement errors of the selected parameters were imposed and their influence on the accuracy of the obtained solution was observed. As a result of the conducted analysis, it appears that errors of all physical parameters applied in the solution are transferred onto the searched value of the heat transfer coefficient. Due to incorrect determinants of the inverse problem, the temperature measurement should be very precise. Also the accuracy of the material data used for computations, such as thermal conductivity and specific heat, is important. It was confirmed by the conducted test calculations. 


\section{Acknowledgements}

The study was performed as part of the regular activity of the AGH University of Science and Technology, Faculty of Metals Engineering and Industrial Computer Science, Work No. 11.11.110.226.

\section{REFERENCES}

[1] A. Gołdasz, Z. Malinowski, T. Telejko, M. Rywotycki, A, Szajding. Archives of Metallurgy and Materials 57 (4), 1143 1149 (2012).

[2] M. Hojny, M. Głowacki, Journal of Engineering Materials and Technology 133 (2), 021008-1-021008-7 (2011).

[3] J. Falkus, K. Miłkowska-Piszczek, Materiali in Tehnologije 49 (6), 903-912 (2015).

[4] Z. Malinowski, J. G. Lenard, M. E. Davies, Journal Of Materials Processing Technology 41 (2), 125-142 (1994).

[5] L.Galdos, E. Saenz de Argandona, J. Mendiguren, R. Ortubay, X.Agirretxe, J. M. Martin, Computer Methods in Materials Science 15 (1), 58-64 (2015).

[6] M. Rosochowska, K. Chodnikiewicz, R. Balendra, Journal of
Materials Processing Technology 145, 207-214 (2004).

[7] M. Rosochowska, R. Balendra, K. Chodnikiewicz, Journal of Materials Processing Technology 135, 204-210 (2003).

[8] C. C. Chang, A. N. Bramley, Proceedings of the Institution of Mechanical Engineers 216 (8), 1179-1186 (2002).

[9] P. Salomonsson, M. Oldenburg, P. Åkerström, G. Bergman, Steel Research Int. 80 (11), 841-845 (2009).

[10] M. Krzyzanowski, J.H. Beynon, Materials Science and Technology, (2015), DOI 10.1179/1743284715Y.0000000125.

[11] Y. Meng, B.G. Thomas, Metallurgical and Materials Transactions B 34 B (5), 685 - 705 (2003)

[12] J. Falkus ( Ed.), Modelling of continuous casting process of steel; 2012 Radom: Wydawnictwo Naukowe Instytutu Technologii Eksploatacji - Państwowy Instytut Badawczy.

[13] M. Rywotycki, Z. Malinowski, A. Szajding, K. Sołek, J. Falkus, K. Miłkowska-Piszczek NHT 2015 : Numerical Heat Transfer 2015 - Eurotherm seminar no. 109 : 27-30 September, Warsaw, Poland 93-94 (2015)

[14] Z. Malinowski, T. Telejko, B. Hadała, A. Cebo-Rudnicka, A. Szajding, International Journal of Heat and Mass Transfer 75, 347-361 (2014).

[15] Z. Malinowski, Numeryczne modele w przeróbce plastycznej i wymianie ciepła. AGH UWN-D, Kraków, (2005). 
